PROCEEDINGS OF THE

AMERICAN MATHEMATICAL SOCIETY

Volume 131, Number 10, Pages 3221-3230

S 0002-9939(03)07165-X

Article electronically published on May 9, 2003

\title{
THE CRITICAL POINT EQUATION ON A THREE-DIMENSIONAL COMPACT MANIFOLD
}

\author{
SEUNGSU HWANG \\ (Communicated by Bennett Chow)
}

\begin{abstract}
On a compact $n$-dimensional manifold $M^{n}$, a critical point of the total scalar curvature functional, restricted to the space of metrics with constant scalar curvature of volume 1, satisfies the critical point equation (CPE), given by $z_{g}=s_{g}^{\prime *}(f)$. It has been conjectured that a solution $(g, f)$ of the CPE is Einstein. Restricting our considerations to $n=3$ and assuming that there exist at least two distinct solutions of the CPE throughout the paper, we first prove that, if the second homology of $M^{3}$ vanishes, then $M^{3}$ is diffeomorphic to $S^{3}$ (Theorem 2). Secondly, we prove that the same conclusion holds if we have a lower Ricci curvature bound or the connectedness of a certain surface of $M^{3}$ (Theorem 3). Finally, we also prove that, if two connected surfaces of $M^{3}$ are disjoint, $\left(M^{3}, g\right)$ is isometric to a standard 3-sphere (Theorem 4).
\end{abstract}

\section{INTRODUCTION}

One main focus in differential geometry has been to find canonical metrics on manifolds. By canonical metrics we mean metrics of constant curvature in various forms. The existence of such metrics on manifolds often has important topological implications. One of the natural ways of finding canonical metrics on smooth manifolds is to look for metrics which are critical points of a natural functional on the space of all metrics on a given manifold. Metrics of constant Ricci curvature, i.e., Einstein metrics, are examples of this approach.

Let $\left(M^{n}, g\right)$ be an $n$-dimensional compact oriented manifold and $\mathcal{M}_{1}$ the set of smooth Riemannian structures on $M^{n}$ of volume 1 . Given a metric $g \in \mathcal{M}_{1}$, let $s_{g}: M^{n} \rightarrow \mathbb{R}$ be its scalar curvature and $d v_{g}$ the volume form determined by the metric and orientation. The total scalar curvature functional $\mathcal{S}: \mathcal{M}_{1} \rightarrow \mathbb{R}$ is defined by

$$
\mathcal{S}(g)=\int_{M^{n}} s_{g} d v_{g}
$$

Einstein and Hilbert showed that the critical points of this functional $\mathcal{S}$ are Einstein metrics. In other words, the critical points satisfy the Euler-Lagrange equation

$$
z_{g}=r_{g}-\frac{s_{g}}{n} g=0
$$

where $r_{g}$ is the Ricci curvature of $g$ and $z_{g}$ is the traceless Ricci tensor.

Received by the editors February 15, 2001.

2000 Mathematics Subject Classification. Primary 53C25.

Key words and phrases. Total scalar curvature functional, critical point, Einstein metric, Fisher-Marsden conjecture. 
On the other hand, due to the resolution of Yamabe's problem, it is known that within each conformal class there exists a metric of constant scalar curvature (csc, hereafter). Thus we may introduce the set of csc-metrics

$$
\mathcal{C}=\left\{g \in \mathcal{M}_{1} \mid s_{g} \text { constant }\right\}
$$

It has been conjectured that the set $\mathcal{C}$ of csc-metrics is rich enough so that the critical points of the total scalar curvature functional $\mathcal{S}$ restricted to $\mathcal{C}$ are also Einstein metrics (Conjecture A).

The Euler-Lagrange equations for a critical point $g$ of this restricted variational problem may be written as the following critical point equation (CPE, hereafter):

$$
z_{g}=s_{g}^{*}(f)
$$

where $f$ is a function on $M^{n}$ with vanishing mean value,

$$
s_{g}^{\prime *}(f)=D_{g} d f-\left(\Delta_{g} f\right) g-f r_{g}
$$

and $\Delta_{g} f=-\frac{s_{g}}{n-1} f$. Here, the operator $s_{g}^{* *}$ is the $L^{2}$ adjoint of the linearization $s_{g}^{\prime}$ of the scalar curvature on $(M, g)$ given by

$$
s_{g}^{\prime}(h)=\Delta_{g} t r h+\delta_{g}^{*} \delta_{g} h-g\left(h, r_{g}\right)
$$

where $h$ is any symmetric bilinear form on $M$ and $\delta$ is the divergence operator.

An immediate observation of the CPE given by (1) is that if $s_{g} /(n-1)$ is not a positive eigenvalue of the Laplacian $\Delta_{g}$, then $g$ is Einstein with $f \equiv 0$. Hence, in terms of the CPE, we may restate our conjecture as follows:

Conjecture A. If there is a non-trivial solution $(g, f)$ of an $n$-dimensional CPE given by (1), then the metric $g$ is Einstein or $f \in K e r s_{g}^{*}$ [1].

J. Lafontaine showed that if a solution metric $g$ of the CPE is conformally flat, such a metric is Einstein 9]. The author showed that if a solution function $f$ of the CPE has its minimum greater than or equal to -1 , then $g$ is Einstein [6]. Bessières-Lafontaine-Rozoy recently showed that if a solution metric $g$ of the CPE is real-analytic, then $g$ is Einstein [2]. The geometric structure of an Einstein solution is known to be simple due to M. Obata, who showed that such a solution is isometric to a standard $n$-sphere [12. Furthermore, we note that if $g$ is an Einstein solution metric of the CPE, then the space of solution functions of the CPE has dimension $n+1$.

This paper is partially motivated by considering the converse of the last statement above. In other words, is the solution metric $g$ isometric to a standard $n$ sphere if there are at least two distinct solution functions of the CPE? It is easy to see that there are at least two distinct solution functions of the CPE if and only if $\operatorname{Ker} s_{g}^{*} \neq 0$, since $\varphi=f_{1}-f_{2} \in \operatorname{Ker} s_{g}^{* *}$ for two solution functions $f_{1}$ and $f_{2}$ of the CPE. It should be noted that if $\operatorname{Ker} s_{g}^{* *}=0$ for some $g$, our conjecture fails. For a special case when $f_{2}=c f_{1}$ for a constant $c \neq 1$, we have $z_{g}=s_{g}^{* *}\left(f_{1}\right)=0$, since $0=s_{g}^{\prime *}(\varphi)=(1-c) s_{g}^{\prime *}\left(f_{1}\right)=(1-c) z_{g}$. The author suggests the following conjecture which claims that this is generally true:

Conjecture B. If Ker $s_{g}^{*} \neq 0$, then $\left(M^{n}, g\right)$ is isometric to a standard $n$-sphere.

The author strongly believes that Conjecture B holds - at least for the case when $n=3$; cf. Remark 1 . In this paper, restricting our considerations to $n=3$, we first investigate the topological aspect of Conjecture B. Note that in [6], we proved 
Theorem 1. Let $f$ be a non-trivial solution of the CPE and $\mu=\inf _{M^{3}}(1+f)<0$. If the Ricci curvature $r_{g}$ satisfies the condition $r_{g}>\frac{1}{\mu} \frac{s_{g}}{3}$, then $H_{2}\left(M^{3}, \mathrm{Z}\right)=0$. In particular, the first Betti number of $M^{3}$ is zero.

For the case when there exist two distinct non-trivial solutions $f_{1}$ and $f_{2}$ of the CPE on $\left(M^{3}, g\right)$, let $\Gamma=\left\{x \in M^{3} \mid \varphi(x)=0\right\}$ for $\varphi=f_{1}-f_{2}$. Then we may prove the following theorem in Section II:

Theorem 2. Assume that $f_{1}$ and $f_{2}$ are two distinct non-trivial solutions of the CPE on $\left(M^{3}, g\right)$. If $H_{2}\left(M^{3}, \mathrm{Z}\right)=0$, then $\Gamma$ is connected and $M^{3}$ is diffeomorphic to $S^{3}$.

As a consequence of Theorems 1 and 2, we have the following.

Corollary 1. Assume that Ker $s_{g}^{* *} \neq 0$. Let $f$ be a non-trivial solution of the CPE on $\left(M^{3}, g\right)$ and $\mu=\inf _{M^{3}}(1+f)$. If $r_{g}>\frac{1}{\mu} \frac{s_{g}}{3}$, then $M^{3}$ is diffeomorphic to $S^{3}$.

It turns out that the converse of Theorem 2 also holds:

Theorem 3. Assume that $f_{1}$ and $f_{2}$ are two distinct non-trivial solutions of the $C P E$ on $\left(M^{3}, g\right)$. If $\Gamma$ is connected, then $M^{3}$ is diffeomorphic to $S^{3}$.

The essential point of Theorems 2 and 3 is that the existence of distinct solutions uniquely determines the topology of $M^{3}$ if there is no embedded compact oriented stable minimal surface in $M^{3}$.

Remark 1. We note that the existence of two distinct solutions is closely related to the Fisher-Marsden conjecture (F-M conjecture, hereafter) as follows. If $f_{1}$ and $f_{2}$ are two distinct non-trivial solution functions of the CPE, then a difference $\varphi=f_{1}-f_{2} \in \operatorname{Ker} s_{g}^{\prime *}$ by (1). Recall that the F-M conjecture states that every solution $(g, \varphi)$ with $\varphi \in \operatorname{Ker} s_{g}^{\prime *}$ is Einstein [3]. Thus, if one could assume the F-M conjecture, the existence of two distinct solutions would imply that $g$ is Einstein. However, this is not true since there exist conformally flat counter-examples of the F-M conjecture [8], 9]. Considering this fact, it is still an interesting open question to ask whether every solution $(g, \varphi)$ with $\varphi \in \operatorname{Ker} s_{g}^{\prime *}$ is conformally flat (Modified F-M conjecture, hereafter). Assuming that the modified F-M conjecture holds, Conjecture B will follow, since every conformally flat solution metric $g$ of the $\mathrm{CPE}$ is Einstein [9] and such an Einstein solution $\left(M^{n}, g\right)$ is isometric to a standard $n$-sphere [12].

In our final Section III, under the assumption that there are two distinct solution functions of the CPE, we show in Lemma 5 that if the set $\Gamma$ is connected, there exists another solution function $\tilde{f}$ of the CPE such that the gradient of $\tilde{f}$ is tangent to $\Gamma$. Then, in the following theorem, we prove that a geometric condition of $\Gamma$ and $\tilde{B}$ uniquely determines the global geometric structure of $M^{3}$, where $\tilde{B}=\tilde{f}^{-1}(-1)$.

Theorem 4. Let $f_{1}$ and $f_{2}$ be two distinct non-trivial solutions of the CPE on $\left(M^{3}, g\right)$. Assume that each of $\Gamma$ and $\tilde{B}$ is connected, and $\Gamma \cap \tilde{B}=\emptyset$. Then $\left(M^{3}, g\right)$ is isometric to a standard 3-sphere.

Remark 2. We note that the set $\Gamma$, the boundary of the nodal domains for the eigenfunction $\varphi$ satisfying $\Delta_{g} \varphi=-\frac{s_{g}}{2} \varphi$, has the following interesting properties. First, there are no critical points of $\varphi$ on $\Gamma$; cf. [3]. Therefore $\Gamma$ is a manifold. In fact, $\Gamma$ is a union of hypersurfaces of $M^{3}$, and $N_{\varphi}=|d \varphi|^{-1} d \varphi$ is defined on 
all of $\Gamma$. Second, it can be shown that at least one connected component of $\Gamma$ is homeomorphic to $S^{2}$; cf. [13] and [6]. Third, $\Gamma$ is totally geodesic, since $D_{g} d \varphi=0$ on $\Gamma$.

\section{The Proofs of Theorems 2 And 3}

This section is devoted to the proofs of Theorems 2 and 3. Throughout the remainder of the present paper, we assume that there exist two distinct solution functions $f_{1}$ and $f_{2}$ of the CPE on $\left(M^{3}, g\right)$. For the proof of Theorem 2, we need the following two topological lemmas.

Lemma 1. Let $N$ be a 3-dimensional orientable compact Riemannian manifold with boundary $\partial N$ such that $\partial N$ is mean convex and has at least one component homeomorphic to $S^{2}$. Then either there is a compact minimal surface $\Sigma$ contained in $N \backslash \partial N$ which is locally of least area, or else $\partial N$ is a 2-sphere and $N$ is diffeomorphic to a closed 3-ball.

Proof. This lemma follows from Proposition 1 (a geometric characterization of handlebodies) and Theorem $1^{\prime}$ (the main existence result for manifolds with mean convex boundary) in [11]. Also, see Lemma 2 in [4].

Let $M_{0, \varphi}=\left\{x \in M^{3} \mid \varphi(x)<0\right\}$, and let $M_{0, \varphi, i}$ be a connected component of $M_{0, \varphi}$ for $i=1, \ldots, k$. Also, let $M_{\varphi}^{0}=\left\{x \in M^{3} \mid \varphi(x)>0\right\}$, and let $M_{\varphi}^{0, j}$ be a connected component of $M_{\varphi}^{0}$ for $j=1, \ldots, k^{\prime}$.

Lemma 2. Either $M_{0, \varphi, i}$ is diffeomorphic to a 3-ball with $S^{2}$ as boundary, or there is a compact stable minimal surface $\Sigma$ in $\overline{M_{0, \varphi, i}}$. The same conclusion holds for $M_{\varphi}^{0, j}$.

Proof. Let $D_{i}=\overline{M_{0, \varphi, i}}$ and $D^{j}=\overline{M_{\varphi}^{0, j}}$ for simplicity. By Remark $2, \partial D_{i}$ is totally geodesic and has at least one component homeomorphic to $S^{2}$. Adding a collar to $D_{i}$ along $\partial D_{i}$, the set $D_{i}$ can be isometrically embedded into a compact Riemannian manifold $\hat{D}_{i}$ with boundary $\partial \hat{D}_{i}$ having the following properties [4]:

(i) each connected component of $\hat{D}_{i}$ is strictly mean convex.

(ii) $\overline{\hat{D}_{i} \backslash D_{i}}$ is diffeomorphic to $[0, \epsilon] \times \partial D_{i}$.

(iii) Any compact minimal surface in $\hat{D}_{i}$ is contained in $D_{i}$.

Here, property (iii) follows from the maximum principle for hypersurfaces, since the level surface $\{t\} \times \partial \hat{D}_{i}$ is convex for each $t \in(0, \epsilon]$. By property (ii), $\partial \hat{D}_{i}$, being diffeomorphic to $\partial D_{i}$, also has at least one component which is homeomorphic to $S^{2}$. Now, applying Lemma 1 to $\hat{D}_{i}=N$, we may conclude that either $\hat{D}_{i}$ is diffeomorphic to a 3-ball with $S^{2}$ as boundary, or else there is a compact stable minimal surface $\Sigma$ in $\hat{D}_{i}$. However, by property (iii), if there is a compact stable minimal surface $\Sigma$ in $\hat{D}_{i}$, such a surface $\Sigma$ must be contained in $D_{i}$. In other words, we may replace $\hat{D}_{i}$ by $D_{i}$, and hence the first statement of the lemma follows, since $M_{0, \varphi, i}$ is diffeomorphic to $\hat{D}_{i}$. The proof for $D^{j}$ is similar.

Now, we are ready to prove Theorem 2.

Proof of Theorem 2. For the proof, we first consider $\check{M}=M^{3} \backslash \Gamma$. Then $\check{M}$ is a disjoint union of $M_{0, \varphi}$ and $M_{\varphi}^{0}$, where $M_{0, \varphi}=\bigcup_{i=1}^{k} M_{0, \varphi, i}$ and $M_{\varphi}^{0}=\bigcup_{j=1}^{k^{\prime}} M_{\varphi}^{0, j}$. Now, we claim that each of $M_{0, \varphi, i}$ and $M_{\varphi}^{0, j}$ is diffeomorphic to 3-balls with $S^{2}$ 
as boundaries. Our claim follows immediately from Lemma 2, if we exclude the existence of the compact oriented stable minimal surface $\Sigma$ in each of $D_{i}$ and $D^{j}$. However, it is well known that each element in $H_{n-1}\left(M^{n}, \mathrm{Z}\right)$ can be represented by sums of embedded compact oriented stable minimal hypersurfaces when $3 \leq n \leq 7$ ([10], p.51). Therefore, our assumption that $H_{2}\left(M^{3}, \mathrm{Z}\right)=0$ implies that there is no embedded compact oriented stable minimal surface $\Sigma$ in $M^{3}$. In particular, there is no such surface $\Sigma$ in each of $D_{i}$ and $D^{j}$. This proves our first claim.

Next, noting that $M^{3}$ can be obtained by gluing $M_{0, \varphi}$ and $M_{\varphi}^{0}$ along their common boundary $\Gamma=\partial M_{0, \varphi}=\partial M_{\varphi}^{0}$, our second claim is that $M_{0, \varphi}$ and $M_{\varphi}^{0}$ are each connected; that is, $k=k^{\prime}=1$. Assume that they are not connected; that is, $k \geq 2$ or $k^{\prime} \geq 2$. Then this assumption leads to the contradiction that $\Gamma$ is not a manifold, since more than two components of $M_{0, \varphi}$ and $M_{\varphi}^{0}$ should be attached together along $\Gamma$ in order to obtain a connected manifold $M^{3}$ after gluing. However, $\Gamma=\varphi^{-1}(0)$ is a manifold, since there are no critical points of $\varphi$ in $\Gamma$ by Remark 2 .

As a consequence of the two claims above, we may conclude that $\Gamma$ is connected and homeomorphic to $S^{2}$. Hence $\overline{M_{0, \varphi}}=M_{0, \varphi} \cup \Gamma$ is a closed 3-ball with $\Gamma \simeq S^{2}$, and so is $\overline{M_{\varphi}^{0}}$. Hence, gluing $\overline{M_{0, \varphi}}$ and $\overline{M_{\varphi}^{0}}$ along $\Gamma \simeq S^{2}$ gives a manifold diffeomorphic to $S^{3}$, since every homeomorphism between two spheres is isotopic to the identity map. This completes the proof of Theorem 2.

The following Lemma 3 is needed in the proof of Theorem 3. This lemma implies that the connectedness of $\Gamma$ determines the topology of $M^{3}$ globally.

Lemma 3. Let $\Gamma$ be connected. Then there is no embedded compact oriented stable minimal surface $\Sigma$ in $\overline{M_{0, \varphi}}$ (or in $\overline{M_{\varphi}^{0}}$ ).

Proof. We prove only the first statement of the lemma - that there is no embedded compact oriented stable minimal surface $\Sigma$ in $\overline{M_{0, \varphi}}$. The proof of the second statement for $\Sigma$ in $\overline{M_{\varphi}^{0}}$ is similar.

Let $\Gamma$ be connected. Now assume that there existed an embedded compact oriented stable minimal surface $\Sigma$ in $\overline{M_{0, \varphi}}$. The first statement of the lemma will be shown by proving that this assumption leads to a contradiction. Under this assumption, the proof consists of the following three contentions.

Contention 1. $\Sigma=\Gamma$.

Proof. Under our assumption, the Laplacian $\Delta_{g}$ and the intrinsic Laplacian $\Delta_{\Sigma}$ on the minimal surface $\Sigma$ are related by

$$
\Delta_{g} \varphi=\Delta_{\Sigma} \varphi+D_{g} d \varphi(\nu, \nu)
$$

where $\nu$ is a normal vector field on $\Sigma$. On the other hand, the equation $s_{g}^{\prime *}(\varphi)=0$ mentioned in Remark 1 is equivalent to

$$
0=D_{g} d \varphi-\left(\Delta_{g} \varphi\right) g-\varphi r_{g}
$$

with $\Delta_{g} \varphi=-\frac{s_{g}}{2} \varphi$, from which we have

$$
D_{g} d \varphi(\nu, \nu)=\varphi r_{g}(\nu, \nu)+\Delta_{g} \varphi
$$

Hence, substitution of (4) into (2) gives

$$
\varphi r_{g}(\nu, \nu)=-\Delta_{\Sigma} \varphi
$$

Let $f$ be $f_{1}$ or $f_{2}$. Then, substitution of $f$ for $\varphi$ into (2) gives

$$
\Delta_{g} f=\Delta_{\Sigma} f+D_{g} d f(\nu, \nu)
$$


and we have

$$
D_{g} d f(\nu, \nu)=(1+f) r_{g}(\nu, \nu)-\frac{s_{g}}{3}+\Delta_{g} f
$$

since we have

$$
(1+f) z_{g}=D_{g} d f+\frac{s_{g} f}{n(n-1)} g
$$

by (1). Thus, substitution of (7) into (6) gives

$$
h r_{g}(\nu, \nu)=-\Delta_{\Sigma} f+\frac{s_{g}}{3}
$$

where $h=1+f$. By (5) and (9), we have

$$
\int_{\Sigma} h \Delta_{\Sigma} \varphi=-\int_{\Sigma} \varphi h r_{g}(\nu, \nu)=\int_{\Sigma} \varphi \Delta_{\Sigma} f-\frac{s_{g}}{3} \varphi .
$$

However, since $\Sigma$ is a manifold without boundary, Green's theorem and Stoke's theorem give

$$
\int_{\Sigma} h \Delta_{\Sigma} \varphi-\varphi \Delta_{\Sigma} f=\int_{\Sigma} \operatorname{div}_{\Sigma}(h d \varphi)-\operatorname{div}_{\Sigma}(\varphi d f)=0 .
$$

Hence, (10) may be reduced to the following equation:

$$
\frac{s_{g}}{3} \int_{\Sigma} \varphi=0
$$

Therefore we may conclude that $\varphi \equiv 0$ on $\Sigma$, since $\Sigma \subset M_{0, \varphi}$ and so $\varphi \leq 0$ on $\Sigma$. This implies that $\Sigma \subset \Gamma=\varphi^{-1}(0)$, and hence $\Sigma=\Gamma$ since $\Gamma$ is connected.

Contention 2. The mean value of $f$ over $\Sigma$ vanishes.

Proof. Since $\Delta_{g} f=-\frac{s_{g}}{2} f$ and $\Delta_{g} \varphi=-\frac{s_{g}}{2} \varphi$, it follows that

$$
\int_{M_{0, \varphi}} f \Delta_{g} \varphi=-\frac{s_{g}}{2} \int_{M_{0, \varphi}} \varphi f=\int_{M_{0, \varphi}} \varphi \Delta_{g} f .
$$

However, we have

$$
\int_{M_{0, \varphi}} f \Delta_{g} \varphi=\int_{\Gamma} f \Phi^{1 / 2}-\int_{M_{0, \varphi}}\langle d f, d \varphi\rangle
$$

where $\Phi=|d \varphi|^{2}$, and

$$
\int_{M_{0, \varphi}} \varphi \Delta_{g} f=\int_{\Gamma} \varphi\left\langle d f, N_{\varphi}\right\rangle-\int_{M_{0, \varphi}}\langle d \varphi, d f\rangle=-\int_{M_{0, \varphi}}\langle d f, d \varphi\rangle .
$$

Therefore, we may conclude from (12), (13), and (14) that

$$
\int_{\Gamma} f \Phi^{1 / 2}=0
$$

On the other hand, $\Phi=|d \varphi|^{2} \neq 0$ by Remark 2 , and moreover $\Phi$ is constant on $\Gamma$ since for any tangent vector $\xi$ to $\Gamma$ we have

$$
\xi(\Phi)=2\left\langle D_{\xi} d \varphi, d \varphi\right\rangle=-s_{g}\langle\xi, d \varphi\rangle \varphi+2 \varphi r_{g}(\xi, \varphi)=0
$$

by (3). Hence, it follows from (15) that

$$
\int_{\Gamma} f=0 \quad \text { or equivalently } \quad \int_{\Sigma} f=0
$$

since $\Sigma=\Gamma$ by Contention 1 . 
Contention 3. The stability condition of $\Sigma$ gives the desired contradiction.

Proof. The stability condition of $\Sigma$ gives

$$
\int_{\Sigma} h^{2}\left(r_{g}(\nu, \nu)+|I I|^{2}\right) \leq \int_{\Sigma}|\nabla h|_{g}^{2}=\int_{\Sigma}|\nabla f|_{g}^{2}
$$

where $|I I|^{2}$ is the length of the second fundamental form of $\Sigma$. However, by (9), we have

$$
\int_{\Sigma} h^{2} r_{g}(\nu, \nu)=\int_{\Sigma}|\nabla f|_{g}^{2}+\frac{s_{g}}{3} h
$$

where $|\cdot|_{g}$ is a norm from the induced metric on $\Sigma$. Also it follows from Contention 1 that $\Sigma=\Gamma$ is totally geodesic, or $|I I|^{2}=0$. Now, substitution of (19) into (18) gives

$$
\int_{\Sigma} h \leq 0
$$

Hence, by (17) and (20), we have

$$
0<\operatorname{Area}(\Sigma)=\int_{\Sigma} 1=\int_{\Sigma} 1+f=\int_{\Sigma} h \leq 0,
$$

which is a contradiction.

Consequently, we may conclude from Contention 3 that there is no compact stable minimal surface $\Sigma$ in $\overline{M_{0, \varphi}}$. This completes the proof of the first statement of Lemma 3.

Now, we are ready to prove Theorem 3.

Proof of Theorem 3. Consider $\check{M}=M^{3} \backslash \Gamma$, where $\check{M}$ is a disjoint union of $M_{0, \varphi}$ and $M_{\varphi}^{0}$. Applying Lemma 3, we may conclude that there is no compact stable minimal surface in $\overline{M_{0, \varphi}}$ (or $\overline{M_{\varphi}^{0}}$ ), and therefore all connected components of $M_{0, \varphi}$ and $M_{\varphi}^{0}$ are diffeomorphic to 3-balls with $S^{2}$ as boundaries by Lemma 2. Gluing $M_{0, \varphi}$ and $M_{\varphi}^{0}$ along their common boundary $\Gamma$ as in the proof of Theorem 2, we have a manifold $M^{3}$ diffeomorphic to $S^{3}$, completing the proof of our theorem.

\section{THE PROOF OF THEOREM 4}

This section is devoted to the proof of Theorem 4. For the proof, we need the following three lemmas.

Lemma 4. For any tangent vector field $X$ to $\Gamma$, we have $z(X, d \varphi)=0$ on $\Gamma$.

Proof. Using (3) and the fact that $\langle d \varphi, X\rangle=0$, we have in a neighborhood of $\Gamma$

$$
\varphi z(d \varphi, X)=\left\langle D_{X} d \varphi, d \varphi\right\rangle=\frac{1}{2}\langle d \Phi, X\rangle
$$

for any vector field $X$ tangent to $\Gamma$. Taking the Lie derivative of (21) with respect to $N_{\varphi}$ on $\Gamma$, we have

$$
\Phi^{1 / 2} z(d \varphi, X)=\frac{1}{2}\left\langle D_{N_{\varphi}} d \Phi, X\right\rangle+\frac{1}{2}\left\langle d \Phi, D_{N_{\varphi}} X\right\rangle .
$$

Note that on $\Gamma$ we have $d \Phi=\frac{1}{2}\left\langle D_{g} d \varphi, d \varphi\right\rangle=0$ since $D_{g} d \varphi=0$ by (3) and

$$
\left\langle D_{N_{\varphi}} d \Phi, X\right\rangle=\left\langle D_{X} d \Phi, N_{\varphi}\right\rangle=X\left\langle d \Phi, N_{\varphi}\right\rangle-\left\langle d \Phi, D_{X} N_{\varphi}\right\rangle=0 .
$$

Hence, the right-hand side of (22) vanishes, and the proof of our lemma follows. 
As a consequence of Lemma 4, we have the following lemma.

Lemma 5. Let $\Gamma$ be connected. Then there exists a solution function $\tilde{f}$ of the CPE such that the gradient of $\tilde{f}$ is tangent to $\Gamma$.

Proof. First we claim that both $\Phi=|d \varphi|^{2}$ and $\eta=\langle d f, d \varphi\rangle$ are constant along $\Gamma$, where $f$ is $f_{1}$ or $f_{2}$. The first statement follows from (16), and the second statement follows from the fact that for any tangent vector field $X$ to $\Gamma$ we have

$$
X(\eta)=\left\langle D_{X} d f, d \varphi\right\rangle+\left\langle d f, D_{X} d \varphi\right\rangle=\left\langle D_{X} d f, d \varphi\right\rangle=(1+f) z(X, d \varphi)=0,
$$

which are the results of $(8)$ and Lemma 4.

Now, let $\tilde{f}=f-\Phi_{0}^{-1} \eta_{0} \varphi$, where $\Phi_{0}=\Phi_{\mid \Gamma}$ and $\eta_{0}=\eta_{\mid \Gamma}$. Then, clearly $\tilde{f}$ is a solution of the CPE, since $s_{g}^{\prime *}(\varphi)=0$. Also, along $\Gamma$ we have

$$
\langle d \tilde{f}, d \varphi\rangle=\langle d f, d \varphi\rangle-\Phi_{0}^{-1} \eta_{0}\langle d \varphi, d \varphi\rangle=\eta_{0}-\eta_{0}=0 .
$$

This implies that the gradient of $\tilde{f}$ is tangent to $\Gamma$, and the proof of Lemma 5 is completed.

Finally, for the solution function $\tilde{f}$ obtained from Lemma 5 , we have

Lemma 6. The following equations hold on $M_{0, \varphi}$ and $M_{\varphi}^{0}$ :

$$
\begin{aligned}
\int_{M_{0, \varphi}}(1+\tilde{f})\left|z_{g}\right|^{2} & =\int_{\Gamma} z\left(d \tilde{f}, N_{\varphi}\right)=-\int_{M_{\varphi}^{0}}(1+\tilde{f})|z|^{2}, \\
\int_{M_{0, \varphi}} \varphi\left|z_{g}\right|^{2} & =\int_{\Gamma} z\left(d \varphi, N_{\varphi}\right)=-\int_{M_{\varphi}^{0}} \varphi\left|z_{g}\right|^{2}
\end{aligned}
$$

where $\Gamma=\partial M_{0, \varphi}=\partial M_{\varphi}^{0}$.

Proof. By (8), the first equality of (23) may be shown by integration by parts in the following way:

$$
\begin{aligned}
\int_{M_{0, \varphi}} & (1+\tilde{f})\left|z_{g}\right|^{2}=\int_{M_{0, \varphi}}(1+\tilde{f}) z_{i j} z^{i j}=\int_{M_{0, \varphi}}\left(D_{g} d \tilde{f}+\frac{s_{g} \tilde{f}}{6} g\right)_{i j} z^{i j} \\
= & \int_{M_{0, \varphi}}\left(D_{g} d \tilde{f}\right)_{i j} z^{i j}=\int_{M_{0, \varphi}} \operatorname{div}(z(d \tilde{f}, \cdot))-\int_{M_{0, \varphi}}(d \tilde{f})_{i}(\delta z)_{k}^{i k}=\int_{\Gamma} z\left(d \tilde{f}, N_{\varphi}\right)
\end{aligned}
$$

where $\delta$ is the codifferential and the last equality comes from Stoke's Theorem and the fact that

$$
\delta z_{g}=\delta\left(r_{g}-\frac{s_{g}}{3} g\right)=\delta r_{g}+d\left(\frac{s_{g}}{3}\right)=\delta r_{g}=-\frac{1}{2} d s_{g}=0 .
$$

The second equality of (23) follows similarly. The equations (24) follow the same method of the proof of (23), since we have

$$
\varphi z_{g}=D_{g} d \varphi+\frac{s_{g} \varphi}{6} g
$$

by (3).

Now, we are ready to prove Theorem 4 .

Proof of Theorem 4. It suffices to prove that $z_{g} \equiv 0$ on $M^{3}=M_{0, \varphi} \cup \Gamma \cup M_{\varphi}^{0}$, since an Einstein solution metric of the CPE is isometric to a standard 3-sphere due to the result in the introduction of Obata ([12]). Let $\check{M}=M^{3} \backslash \Gamma$. Then $\check{M}$ is a disjoint union of $M_{0, \varphi}$ and $M_{\varphi}^{0}$. The proof consists of the following two contentions. 
Contention 1. $z_{g}=0$ on $\Gamma$.

Proof. From the assumption that $\Gamma$ is connected, Lemma 5 implies that there exists a solution function $\tilde{f}$ of the CPE such that the gradient of $\tilde{f}$ is a vector field tangent to $\Gamma$. Then, by Lemma 4 , we have

$$
z\left(d \tilde{f}, N_{\varphi}\right)=\Phi^{-\frac{1}{2}} z(d \tilde{f}, d \varphi)=0
$$

along $\Gamma$.

On the other hand, from the assumption that $\tilde{B} \cap \Gamma=\emptyset$, we have $\tilde{B} \subset \check{M}$. Furthermore, $\tilde{B} \subset M_{0, \varphi}$ or $\tilde{B} \subset M_{\varphi}^{0}$ since $\tilde{B}$ is connected. Without loss of generality, we may assume that $\tilde{B} \subset M_{\varphi}^{0}$. Then, $\tilde{f}$ is strictly greater (or less) than -1 on $M_{0, \varphi}$. Thus the integrand of $\int_{M_{0, \varphi}}(1+\tilde{f})\left|z_{g}\right|^{2}$ is greater (or less) than or equal to 0 . However, applying (25) to the equation (23) gives

$$
\int_{M_{0, \varphi}}(1+\tilde{f})\left|z_{g}\right|^{2}=0
$$

which implies that $z_{g}=0$ on $M_{0, \varphi}$, and, consequently, the continuity of $z_{g}$ implies that $z_{g}=0$ on $\Gamma=\partial M_{0, \varphi}$.

Contention 2. $z_{g}=0$ on $\check{M}=M_{0, \varphi} \cup M_{\varphi}^{0}$.

Proof. By the proof of Contention 1, we have $z_{g}=0$ on $M_{0, \varphi} \cup \Gamma$ if $\tilde{B} \subset M_{\varphi}^{0}$, and $z_{g}=0$ on $M_{\varphi}^{0} \cup \Gamma$ if $\tilde{B} \subset M_{0, \varphi}$. Applying Contention 1 to (24), we have

$$
\int_{M_{0, \varphi}} \varphi\left|z_{g}\right|^{2}=0=-\int_{M_{\varphi}^{0}} \varphi\left|z_{g}\right|^{2}
$$

with $\varphi\left|z_{g}\right|^{2} \leq 0$ on $M_{0, \varphi}$ and $\varphi\left|z_{g}\right|^{2} \geq 0$ on $M_{\varphi}^{0}$. Hence, $z_{g}=0$ on $\check{M}=M_{0, \varphi} \cup M_{\varphi}^{0}$.

Now that we have proved $z_{g} \equiv 0$ on $M^{3}=M_{0, \varphi} \cup \Gamma \cup M_{\varphi}^{0}$ in Contentions 1 and 2 , the proof of the theorem is completed.

\section{REFERENCES}

[1] A.L. Besse, Einstein Manifolds (Springer-Verlag, New York, 1987) MR 88f:53087

[2] Bessières, Lafontaine and Rozoy, Scalar Curvature and Black Holes, Séminaire de Théorie Spectrale et Géométrie, Vol. 18, Année 1999-2000, 69-76, Sémin. Théor. Spectr. Géom., 18, Univ. Grenoble I, Saint-Martin-d'Hères, 2000. MR 2002b:53065

[3] A.E. Fischer and J.E. Marsden, Manifolds of Riemannian Metrics with Prescribed Scalar Curvature, Bull. Am. Math. Soc. 80, 479-484 (1974) MR 49:11561

[4] G. Galloway, On the Topology of Black Holes, Comm. Math. Phys. 151 55-66 (1993) MR 93k:83047

[5] S. Hwang, Critical points and conformally flat metrics, Bull. Korean Math. Soc. 37, No. 3, 641-648 (2000) MR 2001m:58033

[6] S. Hwang, Critical points of the scalar curvature functionals on the space of metrics of constant scalar curvature, Manuscripta Math. 103 135-142 (2000) MR 2001m:58032

[7] S. Hwang, A note on the circle action on Einstein manifold, Bull. Austral. Math. Soc. 63 83-91 (2001) MR 2002e:53061

[8] O. Kobayashi, A differential equation arising from scalar curvature function, J. Math. Soc. Japan 34, No. 4, 665-675 (1982) MR 84a:53046

[9] J. Lafontaine, Sur la géométrie d'une généralisation de l'équation différentielle d'Obata, J. Math. Pures Appliquées 62, 63-72 (1983) MR 84i:53047

[10] H.B. Lawson, Minimal varieties in real and complex geometry (University of Montreal lecture notes, 1974). MR 57:13798

[11] W. Meeks, L. Simon, and S.-T. Yau, Emedded minimal surfaces, exotic spheres, and manifolds with positive Ricci curvature, Ann. Math. 116, 621-659 (1982) MR 84f:53053 
[12] M. Obata, Certain conditions for a Riemannian manifold to be isometric with a sphere, J. Math. Soc. Japan 14, No. 3, 333-340 (1962) MR25:5479

[13] Y. Shen, A note on Fisher-Marsden's conjecture, Proc. Amer. Math. Soc. 125, No.3, 901-905 (1997) MR 97k:53039

Department of Mathematics, Chung-Ang University, 221, HukSuk-Dong, DongJakKu, SEOUl, Korea 156-756

E-mail address: seungsu@cau.ac.kr 